• 研究报告・

\title{
基于最大熵模型的不同尺度物种分布概率优化 热点分析：以红色木莲为例
}

\author{
庄鸿飞 ${ }^{1,2}$ 张殷波 ${ }^{3}$ 王 伟 ${ }^{1,4^{*}}$ 任月恒 ${ }^{1}$ \\ 刘方正 ${ }^{1}$ 杜金鸿 ${ }^{1}$ 周 越 ${ }^{1}$ \\ 1 (国家环境保护区域生态过程与功能评估重点实验室, 中国环境科学研究院, 北京 100012) \\ 2 (山西大学黄土高原研究所, 太原 030006) \\ 3 (山西大学环境与资源学院, 太原 030006) \\ 4 (中国三江并流区域生物多样性协同创新中心, 云南大理 671003)
}

\begin{abstract}
摘要: 单一空间尺度构建的最大熵(maximum entropy, MaxEnt)模型是否具有代表性, 是MaxEnt模型应用与发展中 面临的重要问题。本研究基于有效的地理分布位点数据, 利用最小凸多边形法(the minimum convex polygon method)在三江并流、云南省及全国3个空间尺度下分别识别了红色木莲(Manglietia insignis)的建模区域, 并进一步 建立MaxEnt模型：使用ROC曲线分析法与遗漏率(omission rate, OR)检验评估MaxEnt模型预测精度; 基于ArcGIS 分析分布概率及其热点区域的分布趋势, 并通过分区统计工具Zonal识别潜在适宜分布区域的质心位置; 采用刀 切法检验环境因子贡献率。结果表明: (1)不同尺度下红色木莲的MaxEnt模型都有良好的预测效果, 三江并流、云 南省及全国尺度下的AUC值分别为 $0.936 、 0.887$ 和 0.930 , OR值分别为 $0.18 、 0.15$ 和 0.20 ; (2) 各尺度红色木莲的适生 区格局呈现一致性分布趋势, 集中在独龙江、怒江和澜沧江3个流域; (3) 3 个空间尺度下红色木莲的地理分布受不 同环境因子影响, 存在着尺度依赖效应。由此可见, 红色木莲在不同空间尺度下的预测模型有着稳定的性能表现 与良好的预测效果。此外, 我们建议在野外实地调查与野生生物资源保护中加强对普通物种的关注, 在预测物种 地理分布的研究中将MaxEnt模型与热点分析结合使用。
\end{abstract}

关键词: MaxEnt模型; 空间尺度; 红色木莲; 最小凸多边形; 热点分析; 普通物种

\section{Optimized hot spot analysis for probability of species distribution under different spatial scales based on MaxEnt model: Manglietia insignis case}

\author{
Hongfei Zhuang ${ }^{1,2}$, Yinbo Zhang ${ }^{3}$, Wei Wang ${ }^{1,4^{*}}$, Yueheng Ren ${ }^{1}$, Fangzheng Liu $^{1}$, Jinhong Du$^{1}$, Yue Zhou ${ }^{1}$ \\ 1 State Environmental Protection Key Laboratory of Regional Eco-process and Function Assessment, Chinese Research \\ Academy of Environmental Sciences, Beijing 100012 \\ 2 Institute of Loess Plateau, Shanxi University, Taiyuan 030006 \\ 3 School of Environmental and Resource Sciences, Shanxi University, Taiyuan 030006 \\ 4 Collaborative Innovation Center for Biodiversity and Conservation in the Three Parallel Rivers Region of China, Dali, \\ Yunnan 671003
}

\begin{abstract}
Whether a maximum entropy (MaxEnt) model constructed at one spatial scale is representative of species distributions at other scales is an important issue in the application and development of these models. Using distribution data for Manglietia insignis, we used the minimum convex polygon (MCP) method to model species distribution for three spatial scales—-Three Parallel Rivers, Yunnan Province and China —with a $20 \mathrm{~km}$ buffer outside the distribution region. We built the MaxEnt model for Three Parallel Rivers, Yunnan Province and China using 19, 67, and 88 presence-only records respectively and combined these with data on environmental factors at the point locations. We estimated the prediction accuracy of the MaxEnt model using receiver operating characteristic (ROC) curve and omission rate (OR). Next, we used ArcGIS to analyze
\end{abstract}

收稿日期: 2018-02-13; 接受日期: 2018-05-15

基金项目: 国家重点研发计划(2016YFC0503304)

* 通讯作者 Author for correspondence. E-mail: wang.wei@craes.org.cn 
distribution trends for habitat suitability and potential hotspots. We identified the location of geometric centroid of potentially suitable areas using Zonal and used the Jackknife method to test the dominant environmental factors affecting the distribution of $M$. insignis. We found that the area under ROC curve (AUC value) for Three Parallel Rivers, Yunnan Province and China were 0.936, 0.887, and 0.930 respectively and OR values were 0.18, 0.15, and 0.20, indicating that MaxEnt model for all three spatial scales could successfully predict the distribution of $M$. insignis. Distribution trends of potential habitat suitability and habitat hotspots were consistent between different scales and were concentrated in the river basins of Dulong River, Nujiang River and Lancang River, with no significant zonal transfer for the location of geometric centroid. Different environmental factors affected the geographical distribution of $M$. insignis at the three spatial scales, suggesting scale dependence in the distribution patterns of $M$. insignis. In summary, this study indicates that MaxEnt model of $M$. insignis performs stably and successfully for different spatial scales. In addition, the consistency of results across spatial scales became more obvious for hotspots, indicating that hotspot analysis greatly reduced the effect of spatial scale for the MaxEnt model. Thus, we propose integrating MaxEnt model and hotspot analysis to simulate the geographical distributions of species.

Key words: MaxEnt model; spatial scale; Manglietia insignis; minimum convex polygon; hotspots; common species

深入理解物种的生态特性与地理分布是开展 保护规划与分布预测的重要内容, 也是识别生物多 样性空间格局中生态与进化关键要素的基础(Elith et al, 2011)。国内外学者为寻求一种可以揭示物种环境关系的方法进行了大量探索与研究, 以生态位 理论为基础的物种分布模型 (species distribution models, SDMs) 应运而生, 其概念一经提出便迅速 受到生态学、生物地理学、进化生物学及保护生物 学等科学领域的关注与认可。SDMs模型以生物气 候分析系统(bioclimate analysis and prediction system, BIOCLIM)为倠形, 随着生态学、统计学等理论 的发展、3S技术的推广以及相关研究的推进, 发展 至今, 已涌现出大量的 SDMs 模型, 其中最大熵 (maximum entropy, MaxEnt)模型从中脱颖而出, 成 为目前使用最广的模型(Catherineh et al, 2008; Ahmed et al, 2015; Barbosa \& Schneck, 2015; Vaz et al, 2015)。

MaxEnt 模型是以最大熵原理 (principle of maximum entropy) 为理论支撑的机器学习模型 (Phillips et al, 2006), 基于已有的、数量有限的物种 分布点数据以及分布点对应的环境条件, 构成未知 分布的约束条件, 建立物种地理分布与环境因子间 的联系, 通过特定算法找出物种分布规律的最大熵, 进行未知区域的物种分布概率预测(分布的适宜程 度) (Phillips et al, 2004)。自Phillips (2006)等首次引 入MaxEnt模型预测物种潜在分布之后, 它已成为 生态学研究的热点工具。
近年来, 国内外有关研究开始关注不同条件下 MaxEnt模型性能的稳定性与不确定性(尚忠慧, 2016) ${ }^{\circledR}$, 主要集中于数据质量和参数设置两方面对 MaxEnt模型预测精度及输出结果的影响, 如: 不同 分辨率的环境图层下预测精度的比较(Song et al, 2013); MaxEnt模型预测效果对分布位点数量的响 应(陈新美等, 2012); 不同来源的分布位点数据下 MaxEnt模型预测精度与潜在适宜分布区域的一致 性探讨(Lin et al, 2015); MaxEnt模型在不同阈值选 择下预测物种分布的表现(Escalante et al, 2013); 物 种分布数据不充分的条件下最优参数的设置 (Morales et al, 2017); MaxEnt模型复杂度对物种潜 在分布区预测的影响(朱耿平和乔慧捷, 2016)。尺度 与格局是生态学研究的核心问题, 也是统一种群生 物学与生态系统科学、综合基础生态学与应用生态 学的关键(Levin, 1992)。MaxEnt模型对空间尺度变 化的适用能力是其进一步发展与应用的重要制约 因素, 目前有关MaxEnt模型的研究仍停留在单一 空间尺度, 关于MaxEnt模型性能与空间尺度的关 系研究较少。

鉴于此, 本研究以红色木莲(Manglietia insignis) 为研究对象, 以三江并流、云南省和全国的行政范 围为基础构建 3 个尺度的建模区域, 分别建立 MaxEnt模型, 开展以下三方面的研究: (1)检验红色 木莲MaxEnt模型在3个尺度下预测精度的稳定性;

(1) 尚忠慧 (2016) 基于 Maxent 的物种空间分布预测不确定性分析以当归为例. 硕士学位论文, 陕西师范大学, 西安. 
(2)分析不同尺度下红色木莲分布概率的空间格局 变化; (3)研究基于MaxEnt模型建立的红色木莲物种 一环境关系的尺度效应, 旨在探讨红色木莲的潜在 分布预测对建模尺度变化的响应, 为MaxEnt模型 的更好应用提供理论支持。

\section{材料与方法}

\section{1 研究对象与尺度设置}

\subsection{1 研究对象概况}

红色木莲是木兰科木莲属 (Manglietia) 常绿乔 木, 为IUCN红色名录无危等级(Least Concern, LC) 物种, 《中国生物多样性红色名录: 高等植物卷》 中列为易危(VU)物种 (http://www.zhb.gov.cn/gkml/ hbb/bgg/201309/W020130917614244055331.pdf)。红 色木莲生长于海拔1,700-2,500 m的常绿阔叶林或常 绿落叶阔叶混交林中, 喜温凉湿润气候。其分布范围 广阔，涵盖了我国湖北、湖南、福建、广西、四川、 贵州、云南和西藏等 8 个省份，尼泊尔、印度和缅甸 等国也有分布(中国科学院昆明植物研究所，2006; 董学芬, 2017)。

\subsection{2 尺度设置}

本研究最小尺度是三江并流尺度, 参照联合国 教科文组织世界遗产委员会批准的云南三江并流 世界自然遗产地确定范围, 包括了丽江市、迪庆藏 族自治州和怒江傈僳族自治州所涵盖的 8 个市县的 行政范围(http://whc.unesco.org/en/list/1083)。该区域 位于全球生物多样性热点地区一一西南山地的核
心地带，其内的云南三江并流保护区符合世界自然 遗产地全部入选标准, 具有突出普遍价值(outstanding universal value)。中间尺度为云南省，位于我国 的西南边陲，是我国生物多样性最为丰富的省份之 一。最大尺度为全国尺度。尺度设置详见表 1 。

基于3个空间尺度下可获得的有效地理分布信 息，利用ArcGIS 10.2中最小边界几何 (minimum bounding geometry)工具分别构建红色木莲分布位 点的最小凸多边形(minimum convex polygon, MCP), 得到红色木莲可能分布的区域范围。红色木莲主要 由鸟类传播种子(柴勇等, 2011), 鸦科鸟是常见的植 物种子扩散者, 扩散距离通常小于 $5 \mathrm{~km}$, 其中星鸦 (Nucifraga caryocatactes)传播种子的距离记录达20 km (蒋志刚, 1996; 李宏俊和张知涁, 2001)。本研究 在各尺度 MCP分布范围的基础上，利用 ArcGIS 10.2中的缓冲区(buffer)工具向外扩张20 km, 以此 作为各尺度的建模区域(图1)。

\section{2 研究方法}

\subsection{1 数据收集与处理}

仅采用红色木莲的自然分布位点数据, 主要来 源于：中国数字植物标本馆(http://www.cvh.org.cn/) 的标本记录; 全球生物多样性信息网络(GBIF, https://www.gbif.org/)的分布记录; 学术论文、科考报 告、专著等相关文献资料中红色木莲的地理分布信 息。剔除精度不足的地理分布位点后，全国范围内共 得到88个有效位点，涵盖了湖南、广西、四川、贵州、 云南、西藏等红色木莲在我国的主要分布区，其中三

表1 研究区域的尺度设置与红色木莲的分布现状

Table 1 Scale setting of the study areas and the distribution status of Manglietia insignis

\begin{tabular}{|c|c|c|}
\hline $\begin{array}{l}\text { 尺度 } \\
\text { Scale }\end{array}$ & $\begin{array}{l}\text { 覆盖范围 } \\
\text { Cover range }\end{array}$ & $\begin{array}{l}\text { 红色木莲分布现状 } \\
\text { Distribution status of Manglietia insignis }\end{array}$ \\
\hline $\begin{array}{l}\text { 三江并流 } \\
\text { Three Parallel } \\
\text { Rivers }\end{array}$ & $\begin{array}{l}\text { 泸水县、兰坪白族普米族自治县、玉龙纳西族自治县、福贡县、维西 } \\
\text { 傈僳族自治县、德钦县、贡山独龙族怒族自治县和香格里拉市 } 8 \text { 个行 } \\
\text { 政县, 约 } 4.5 \text { 万 } \mathrm{km}^{2} \\
\text { Including Lushui County, Lanping Bai Pumi Autonomous County, Yulong } \\
\text { Naxi Autonomous County, Fugong County, Weixi County, Deqin County, } \\
\text { Gongshan County, and Shangri-La City, about } 45,000 \mathrm{~km}^{2}\end{array}$ & $\begin{array}{l}\text { 泸水县、福贡县、德钦县和贡山独龙族怒族自治 } \\
\text { 县有其分布记录 } \\
\text { Lushui County, Fugong County, Deqin County, and } \\
\text { Gongshan County with distribution records }\end{array}$ \\
\hline $\begin{array}{l}\text { 云南省 } \\
\text { Yunnan Province }\end{array}$ & $\begin{array}{l}\text { 包含昆明市、玉溪市和迪庆藏族自治州等 } 16 \text { 个州市, 约39万 } \mathrm{km}^{2} \\
\text { Containing } 16 \text { cities, such as Kunming, Yuxi, and Dêqên Tibetan Autono- } \\
\text { mous Prefecture, about } 390,000 \mathrm{~km}^{2}\end{array}$ & $\begin{array}{l}\text { 临沧市、普洱市和玉溪市等12个州市有其分布 } \\
\text { 记录 } \\
\text { Twelve cities including Lincang City, Puer City, and } \\
\text { Yuxi with distribution records }\end{array}$ \\
\hline $\begin{array}{l}\text { 全国 } \\
\text { Country }\end{array}$ & $\begin{array}{l}\text { 包含省、自治区、直辖市及特别行政区在内的 } 34 \text { 个省级行政区域, 约 } \\
960 \text { 万 } \mathrm{km}^{2} \\
\text { Containing } 34 \text { provincial administrative regions, including provinces, } \\
\text { autonomous regions, municipalities, and special administrative regions, } \\
\text { about } 9,600,000 \mathrm{~km}^{2}\end{array}$ & $\begin{array}{l}\text { 湖北、湖南、福建、广西、四川、贵州、云南和 } \\
\text { 西藏8省有其分布记录 } \\
\text { Hubei, Hunan, Fujian, Guangxi, Sichuan, Guizhou, } \\
\text { Yunnan, Tibet with distribution records }\end{array}$ \\
\hline
\end{tabular}




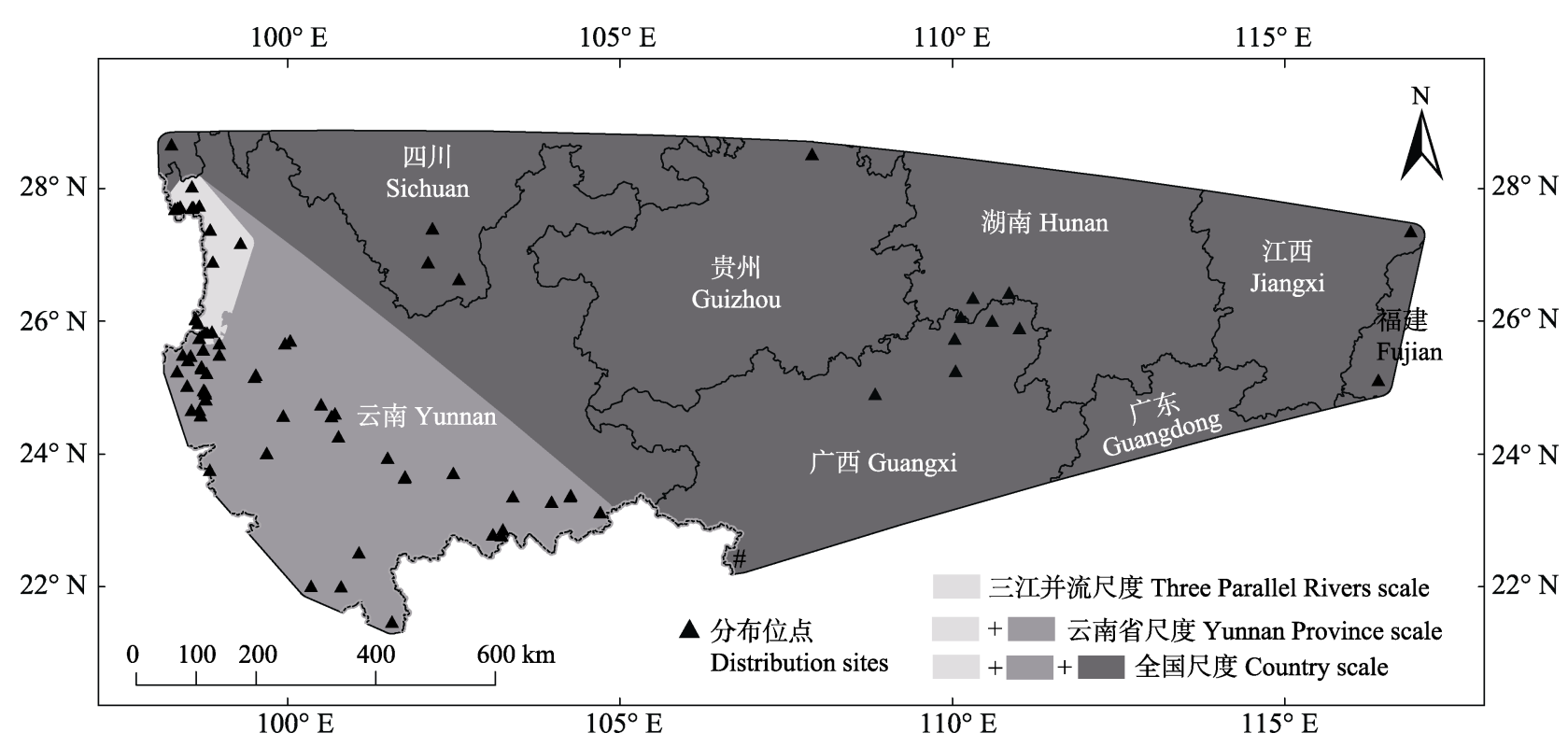

图13个空间尺度的建模区域与红色木莲分布位点

Fig. 1 Modeling areas of three spatial scales and distribution sites of Manglietia insignis

江并流、云南省尺度分别包含19个和67个分布位点。 按照模型软件MaxEnt 3.3.3k (http://www.cs.princeton. edu/schapire/Maxent/)要求, 分别生成csv格式的输 入文件。

气候与地形因子是植物群落形成与发展过程 中极为重要的环境因子(陈飞等, 2012)。本研究将19 个生物气候因子与 3 个地形因子作为环境因子, 各 环境因子数据描述及来源等信息详见附录 1 。由于 环境因子数据的来源不一，其尺度范围、坐标信息、 栅格分辨率等属性信息各不相同, 为保证MaxEnt 模型顺利运行, 基于ArcGIS 10.2对22个环境因子图 层进行预处理: 统一地理坐标系至WGS 1984; 数 据的栅格分辨率重采样至 $30 "$ (约为 $1 \mathrm{~km}$ ); 分别提 取三江并流、云南省与全国3个尺度建模区域的环 境因子图层。

为消除环境因子多重共线性对建模的不利影 响(Nüchel et al, 2018), 利用R 3.4.3的psych包分别 对 3 个尺度下建模区域的生物气候因子与地形因子 数据进行Pearson相关性分析, 笁选出相关系数低于 0.7的环境因子(Manhães et al, 2018)。三江并流尺度 参与建模的环境因子为: 年平均温度(b1)、昼夜温 差月均值(b2)、温度季节变化标准差(b4)、降水量变 异系数(b15)、海拔(alt)、坡度(slo)、坡向(asp)。云 南省尺度: 年平均温度(b1)、昼夜温差月均值(b2)、 温度季节变化标准差(b4)、年均降水量(b12)、最干 月降水量(b14)、降水量变异系数(b15)、海拔(alt)、 坡度(slo)、坡向(asp)。全国尺度: 年平均温度(b1)、 昼夜温差月均值(b2)、年均温度变化范围(b7)、年均 降水量(b12)、最暖季度降水量(b18)、海拔(alt)、坡 度(slo)、坡向(asp)。将环境因子数据转化为ASCII 格式, 作为MaxEnt 3.3.3k中环境图层(environmental layers)的输入文件。

\subsection{2 数据分析}

在MaxEnt 3.3.3k中导入处理好的地理分布位 点数据与环境因子数据, 然后设置模型参数。根据 地理分布位点数量, 三江并流尺度与云南省尺度的 建模选择线性特征(linear features)、二次型特征 (quadratic features)与片段化特征(hinge features)的 特征组合, 全国尺度建模选择线性、二次型特征、 乘积型特征(product features)、阈值性特征(threshold features)与片段化特征(Elith, 2011), 并选择10\%存 在点训练集逻辑斯蒂 (10\% training presence)作为各 尺度模型的应用阈值规则(Raes et al, 2009); 并将 MaxEnt模型重复运行次数设为 20 次, 以消除随机 性(张路, 2015a, b), 其他参数保持默认值。模型输出 结果为一系列表示红色木莲分布概率的栅格。

使用受试者工作曲线(receiver operating characteristic, ROC) 与遗漏率 (omission rate, OR) 检验 MaxEnt模型预测精度, 并通过比较3个空间尺度下 MaxEnt的ROC曲线下面积 (area under the curve, 
AUC)与 OR值评估模型预测精度的稳定性(Phillips et al, 2006)。AUC值在0-1之间, 值越大, 模型预测 精度越高, 一般认为AUC值 > 0.7时, MaxEnt模型 的预测结果便为可信(Raes \& Steege, 2007); OR值 越小MaxEnt预测精度越高(Phillips et al, 2006)。

基于三江并流尺度的建模区域分析各尺度下 红色木莲适生区的空间格局，主要从两个方面进行 研究: (1)分布概率的空间一致性分析。为了深入揭 示分布概率的空间格局对尺度选择的响应规律, 本 研究基于ArcGIS 10.2对MaxEnt模型输出的概率值 信息进行符号化展示, 并使用优化热点分析工具 (optimized hot spot analysis)进一步对MaxEnt模型输 出的分布概率进行归一化处理, 识别并分析 3 个尺 度下红色木莲概率分布的热点区域。(2)潜在适宜分 布区域质心位置分析。本研究使用分区统计工具 Zonal分别计算3个空间尺度下潜在适宜分布区域的 几何质心(centroid), 通过质心位置的变化表征红色 木莲空间分布的变迁(唐继洪等, 2017)。综合分布概 率的空间一致性与质心位置的分析结果, 判断不同 空间尺度下红色木莲适生区的空间格局变化。

本研究采用刀切法(Jackknife)检验环境因子贡 献率(胡秀等, 2014), 基于此识别影响红色木莲空间 分布的主导环境因子。本研究选择贡献率大于 $20 \%$ 的环境因子作为主导环境因子, 进一步比较 3 个空 间尺度下主导环境因子的异同，分析MaxEnt模型 建立的物种-环境关系的尺度效应。

\section{2 结果}

\subsection{MaxEnt模型预测精度}

MaxEnt模型重复运行20次后得到的ROC曲线
如图2所示，三江并流、云南省和全国3个空间尺度 下的AUC平均值分别为 $0.936 \pm 0.015 、 0.887 \pm 0.010$ 和 $0.930 \pm 0.014$; 各尺度的 $\mathrm{OR}$ 值分别为 $0.18 \pm$ $0.092 、 0.15 \pm 0.035$ 和 $0.20 \pm 0.045$ 。AUC值与 OR值 的检验结果都表明各尺度建立的MaxEnt模型都有 良好的预测效果。

\section{2 红色木莲适生区空间格局}

如图3所示，3个尺度下红色木莲适宜性概率的 分布趋势基本一致，高值概率都集中在独龙江、怒 江、澜沧江3个流域。3个空间尺度下的热点区域(极 显著热点区域十显著热点区域十热点区域)集中分 布趋势更加明显(图4)。从行政区域上看，除兰坪白 族普米族自治县外，三者热点区域的空间分布格局 保持高度一致。从 3 个潜在适宜分布区域的质心位置 来看(图3), 不同尺度下的几何质心都分布在怒江流 域周围，通过ArcGIS 10.2的点距离(point distance) 分析工具可得，三江并流尺度质心到云南、全国尺度 质心的距离分别为 $15.59 \mathrm{~km}$ 和31.95 km, 云南尺度质 心到全国尺度质心的距离为 $17.01 \mathrm{~km}$, 与研究区域(三 江并流 4.5 万 $\mathrm{km}^{2}$ ，云南省 39 万 $\mathrm{km}^{2}$ )相比，3个尺度质 心在空间位置上分布集中，可视为无地带性转移。

\section{3 环境因子贡献率}

3 个空间尺度下环境因子贡献率的检验结果如 表2、图5。三江并流尺度下，海拔(39.4\%)和坡向 (22.1\%) 是影响红色木莲地理分布的主导因子; 云 南省尺度下，年均降水量(35.5\%)是影响红色木莲 地理分布的主导因子; 全国尺度下，昼夜温差月均 值(24.8\%)和年均温度变化范围(21.7\%)是影响红色 木莲地理分布的主导因子。
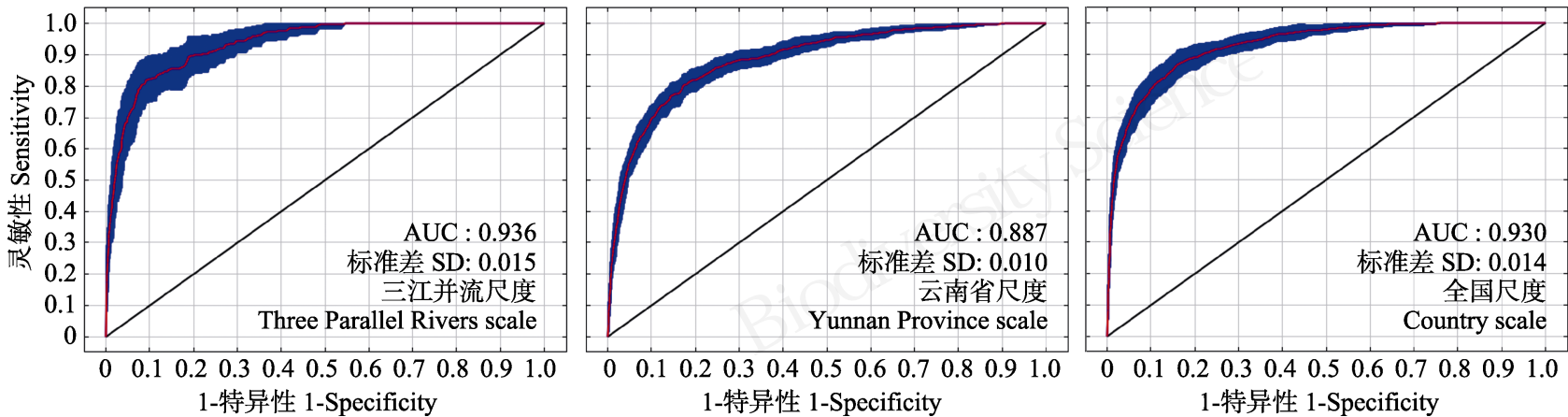

图2 3个空间尺度的受试者工作曲线与曲线下面积

Fig. 2 Receiver operating characteristic curve of three spatial scales and area under the curve (AUC) 
$\mathbf{A}$

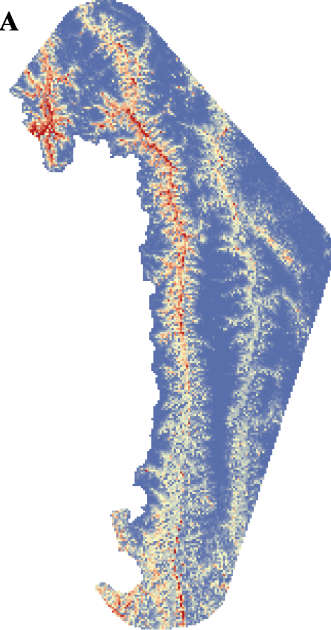

三江并流尺度

Three Parallel Rivers scale

0.91

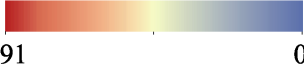

B

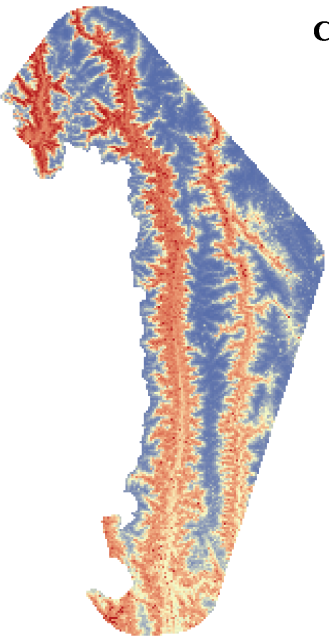

云南省尺度

Yunnan Province scale

0.97
C

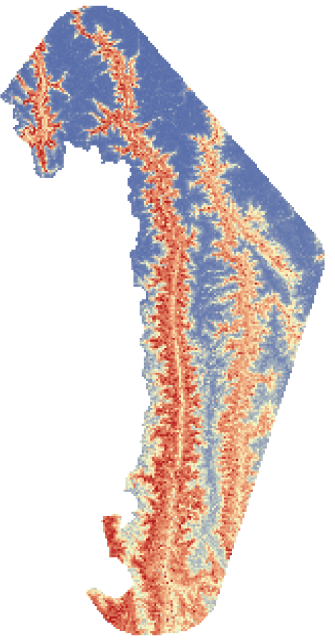

全国尺度

Country scale

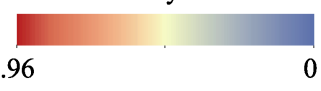

$\begin{array}{lllll}0 & 25 & 50 & 100 & 150 \mathrm{~km}\end{array}$

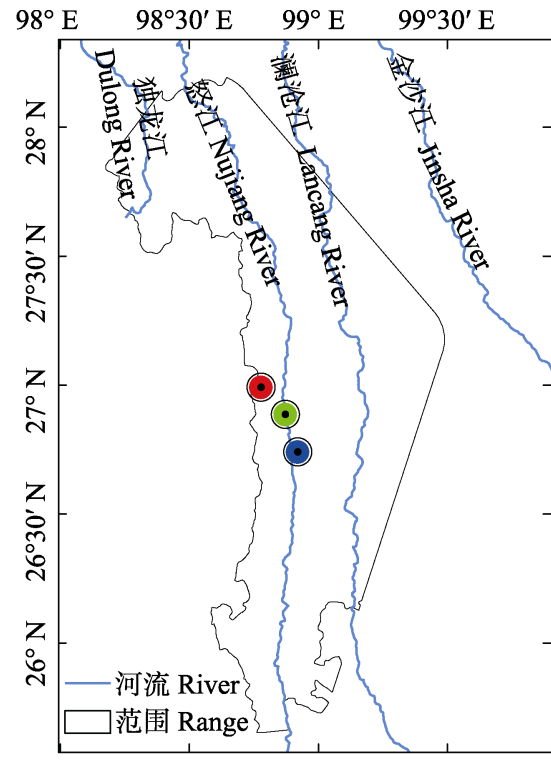

○三江并流尺度质心

Three Parallel Rivers scale centroid ○云南尺度质心

Yunnan Province scale centroid

○全国尺度质心

Country scale centroid

图3 3个空间尺度下红色木莲的潜在适宜性及其质心分布

Fig. 3 Potential suitable distributions and its centroids of Manglietia insignis under three spatial scales

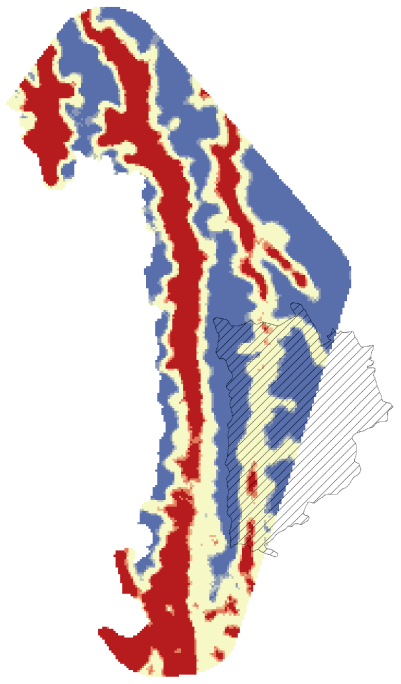

三江并流尺度

Three Parallel Rivers scale

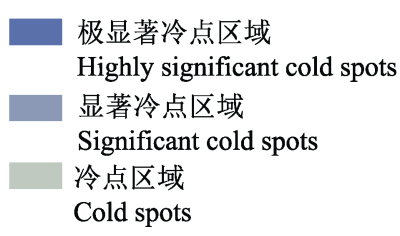

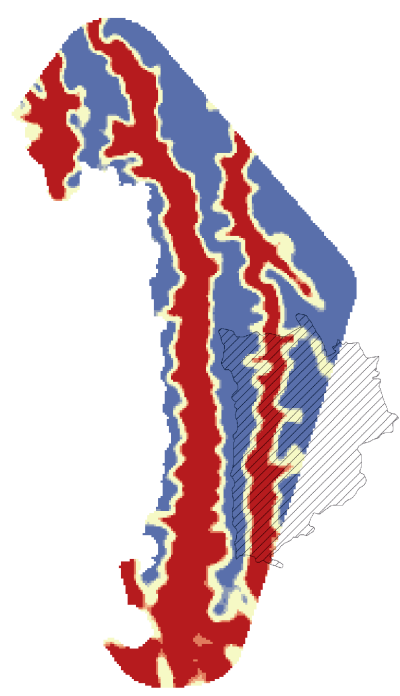

云南省尺度

Yunnan Province scale

热点区域

Hot spots

显著热点区域

Significant hot spots

极显著热点区域

Highly significant hot spots

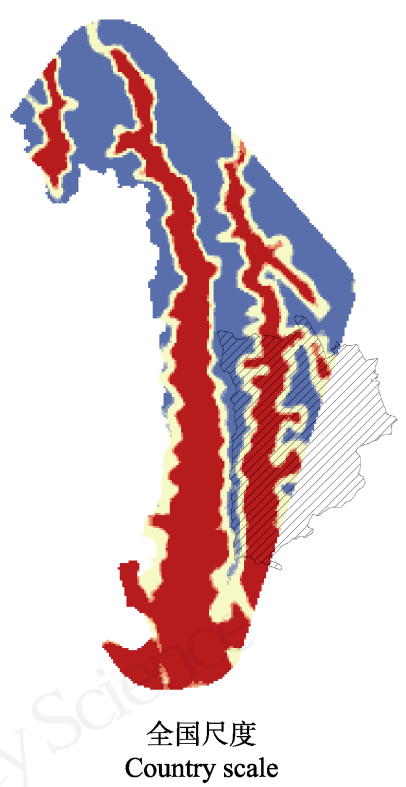

离散单元

Discrete elements

兰坪白族普米族自治县

Lanping Bai and Pumi Autonomous County

$0 \quad 37.5 \quad 75.0$ $150 \mathrm{~km}$

图4 3个空间尺度下红色木莲分布概率的优化热点分析

Fig. 4 Optimized hot spot analysis of probability distribution of Manglietia insignis under three spatial scales 
表2 三江并流、云南省、全国3个尺度下红色木莲MaxEnt模型的环境因子贡献率

Table 2 Contribution of environmental variables for Manglietia insignis under Three Parallel Rivers scale (TPRS), Yunnan Province scale (YPS), and country scale (CS)

\begin{tabular}{|c|c|c|c|c|c|}
\hline $\begin{array}{l}\text { 尺度 } \\
\text { Scale }\end{array}$ & $\begin{array}{l}\text { 变量 } \\
\text { Variable }\end{array}$ & $\begin{array}{l}\text { 环境因子贡献率 } \\
\text { Contribution (\%) }\end{array}$ & $\begin{array}{l}\text { 尺度 } \\
\text { Scale }\end{array}$ & $\begin{array}{l}\text { 变量 } \\
\text { Variable }\end{array}$ & $\begin{array}{l}\text { 环境因子贡献率 } \\
\text { Contribution (\%) }\end{array}$ \\
\hline \multirow{7}{*}{$\begin{array}{l}\text { 三江并流尺度 } \\
\text { TPRS }\end{array}$} & b1 & 3.4 & \multirow{12}{*}{$\begin{array}{l}\text { 全国尺度 } \\
\text { CS }\end{array}$} & b15 & 13.7 \\
\hline & b2 & 9.8 & & alt & 2.9 \\
\hline & b4 & 6.2 & & slo & 10.1 \\
\hline & b15 & 11 & & asp & 3.5 \\
\hline & alt & $39.4^{*}$ & & b1 & 5.9 \\
\hline & slo & 8.1 & & b2 & $24.8^{*}$ \\
\hline & asp & $22.1^{*}$ & & b7 & $21.7^{*}$ \\
\hline \multirow{5}{*}{$\begin{array}{l}\text { 云南省尺度 } \\
\text { YPS }\end{array}$} & b1 & 5.1 & & b12 & 11.4 \\
\hline & b2 & 8.9 & & b18 & 14.1 \\
\hline & b4 & 18.8 & & alt & 7.1 \\
\hline & b12 & $35.5^{*}$ & & slo & 10.4 \\
\hline & b14 & 1.6 & & asp & 4.7 \\
\hline
\end{tabular}

* 表示主导环境因子。变量的描述参见附录 1 。

* The dominant environmental factors. The description of variables is shown in Appendix 1.
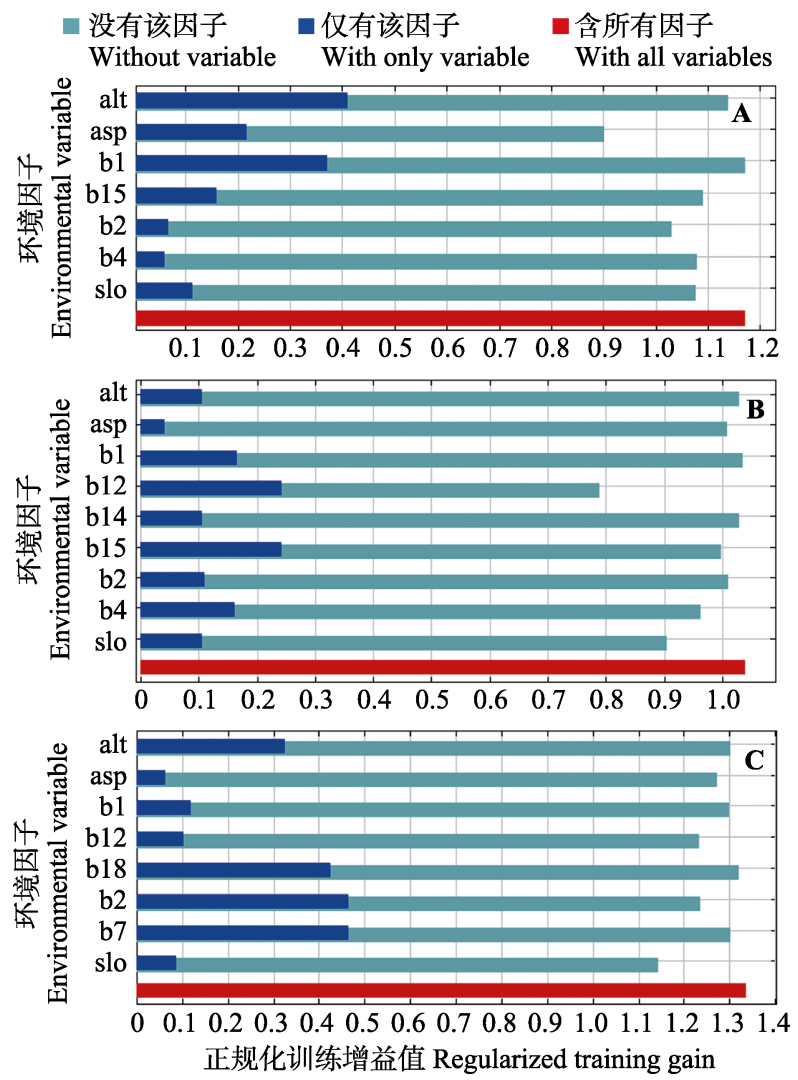

图5 三江并流尺度 $(\mathrm{A}) 、$ 云南省尺度(B)与全国尺度(C)下环 境因子正规化训练增益值的刀切法评估

Fig. 5 Results of Jackknife evaluation of the environmental variables with respect to regularized training gain for Three Parallel Rivers scale (A), Yunnan Province scale (B), and country scale (C)

\section{3 讨论}

本研究基于有效的地理分布位点, 构建三江并 流、云南省与全国尺度下红色木莲的建模区域, 并 通过相关性检验篮选出各尺度下参与建模的环境 因子数据, 在 3 个空间尺度下建立了红色木莲的 MaxEnt模型, 探索红色木莲的潜在分布预测对空 间尺度的响应情况。综合模型预测精度评估与空间 分布格局分析, 红色木莲的MaxEnt模型在不同空 间尺度下有着稳定的性能表现。一定数量与质量的 物种地理分布位点是构建高预测精度MaxEnt模型 的基础(陈新美, 2012), 但在实际研究中常常面临分 布位点数据缺乏的问题, 导致无法建立生态位完整 的MaxEnt模型, 预测结果可能会过低估计物种的 适生区分布范围。空间尺度的扩大意味着可以获取 更多的物种信息, 为建立系统全面的物种-环境关 系提供更为丰富的环境信息。本研究结果表明更大 尺度(云南省和全国尺度)的MaxEnt模型同样可以有 效预测物种在局部地区(三江并流尺度)的适生区分 布, 通过空间尺度的转换解决基础数据收集困难的 问题, 为MaxEnt模型在不同空间尺度的应用提供 了新思路。

3 个空间尺度下红色木莲适生区分布格局在大 部分区域呈现出一致性, 但在兰坪白族普米族自治 
县出现差异。通过查阅此地的科考报告、专著及学 术论文等文献资料, 我们发现其境内野生生物资源 的本底调查仍不全面, 物种名录及其种群数量尚不 明确(杨国斌, 2011, 2013; 汪海林, 2017)。红色木莲 在该区域分布位点的缺失极有可能是由于野外调 查与保护的缺位, 进而导致了本研究三江并流尺度 下MaxEnt模型的预测结果在此区域的空白。考虑到 保护一个物种的最佳时期是当它处于普通物种状 态的时候(Scott et al, 1987), 本研究选择红色木莲这 一普通物种作为研究对象, 分析结果反映出过去实 地野外调查与野生生物资源保护往往偏向关注面 临灭绝风险、生境狭窄的物种及其生境, 而较少考 虑普通物种, 并且这种 “只抓重点”的保护模式对普 通物种野生资源构成的威胁已经开始显现，2013年 红色木莲被列为 《中国生物多样性红色名录: 高等 植物卷》VU等级物种。因此, 本研究建议在兰坪白 族普米族自治县开展滇金丝猴(Rhinopithecus bieti)、 怒江金丝猴(R. strykeri)等关键物种专项保护的同时, 也进一步加强针对以红色木莲代表的普通物种的 调查研究, 将普通物种的保护纳入兰坪白族普米族 自治县的保护体系当中。

不确定性是影响决策的重要因素(Myers, 1990), 空间尺度作为不确定性因素对MaxEnt模型进一步 的应用与发展有明显的限制作用。本研究尝试使用 热点分析的方法将MaxEnt模型输出结果进行处理, 探究热点分析在预测物种适生区方面的作用。结果 显示, 热点区域(图4)比MaxEnt模型的直接预测结 果(图3)更加清晰地呈现了红色木莲的适生区空间 格局，并且不同尺度间的一致性更加明显，可见热 点分析在很大程度上能消除空间尺度给MaxEnt模 型带来的不确定性影响。最小存活种群理论表明物 种以一定概率存活下去需要基于有效种群大小、最 小存活面积等条件, 可见破碎化生境不利于物种的 长期生存。热点分析可以有效探测研究区域内具有 显著统计学意义的局部热点区域(Getis \& Ord, 1992; Ord \& Getis, 1995), 除关注分布概率的高低, 更加 强调地理单元间的位置关系, 大大削弱了红色木莲 适生区分布的破碎化程度, 更能反映其真实分布状 态，并且边界成本的降低对保护资源的高效利用与 自然保护地的合理布局具有重要意义。我们相信综 合MaxEnt模型的预测功能与热点分析的聚类优化 将十分有益于物种地理分布的科学研究与保护规
划的开展。

环境因子贡献率的刀切法检验结果表明, 不同 空间尺度下红色木莲的地理分布受不同的环境因 子所影响, 地形因子在较小空间尺度内(三江并流 尺度)有重要影响，而转移到较大空间尺度下(云南 省与全国尺度)其作用则不显著。海拔梯度反映了温 度和湿度等环境因子的综合效应，对山地物种分布 规律的研究具有重要意义(唐志尧和方精云, 2004)。 三江并流区域位于西南山地，海拔分布从 $760 \mathrm{~m}$ (怒 江河谷)到6,740 m (卡瓦格博峰), 狭小地域内的巨 大高差使该区域内的物种分布对海拔梯度变化十 分敏感，这也是海拔和坡向等地形因子主导该尺度 下红色木莲分布的重要原因。随着空间尺度的扩大, 除海拔等地形因子信息外，其他环境因子信息也丰 富起来, 且红色木莲分布位点的数量增多, 为 MaxEnt模型的建立提供了更多的约束条件，年均 降水量、昼夜温差月均值和年均温度变化范围等生 物气候因子对红色木莲分布的影响突显出来。由此 可见，红色木莲的空间分布格局存在着尺度依赖效 应，特定尺度下的空间格局可能存在特定主导因子 (Wiegand et al, 2007), 因此在挑选参与建模的环境 因子时应充分考虑空间尺度对物种分布的影响。

物种的地理分布是不均匀的(赵淑清等，2000), 弄清区域内物种资源的分布现状是实施精细化保 护的关键。根据本研究结果, 红色木莲在兰坪白族 普米族自治县和维西傈僳族自治县均有大面积分 布，但这两个区域却鲜有此种的分布记录(表1)。可 能有两方面原因导致记录的缺失：一是三江并流区 域内环境异质性极大，沟谷纵横与气候多变的环境 条件让基础性研究工作的开展十分困难; 二是三江 并流区域内的保护资源与人员有限，物种资源调查 工作仅针对重点保护物种, 无法全面铺开。本研究 表明，基于MaxEnt模型通过扩大空间尺度的方法 可以有效克服基础性研究工作不足，并且得到的预 测结果可以为下一步的野外调查研究提供新样地, 实现模型预测与实地调查工作的良性循环。三江并 流区域是世界生物多样性热点区域, 如果等数据收 集充分再开展精细化保护, 很多物种可能已经灭绝 (Sierra et al, 2002), 因此我们建议基于已有的物种 信息利用MaxEnt模型等相关方法预测三江并流区 域内更多物种的地理分布，随后结合实地考察逐步 掀开该区域生物多样性的神秘面纱，使三江并流区 
域生物多样性的精细化保护在不断探索与实践中 得到完善。

\section{参考文献}

Ahmed SE, McInerny G, O’Hara K, Harper R, Salido L, Emmott S, Joppa LN (2015) Scientists and software-surveying the species distribution modelling community. Diversity and Distributions, 21, 258-267.

Barbosa FG, Schneck F (2015) Characteristics of the top-cited papers in species distribution predictive models. Ecological Modelling, 313, 77-83.

Catherineh G, Jane E, Robertj H, Antoine G, Townsend PA, Bettea L (2008) The influence of spatial errors in species occurrence data used in distribution models. Journal of Applied Ecology, 45, 239-247.

Chai Y, Zhu H, Meng GT, Shi JP, Yang GB (2011) Population structure and distribution pattern of dominant tree species in ancient tea tree community in Ailao Mountains of Yunnan Province, China. Forest Research, 24, 277-284. (in Chinese with English abstract) [柴勇, 朱华, 孟广涛, 施济普, 杨国 平 (2011) 云南哀牢山古茶树群落优势树种的种群结构 与分布格局. 林业科学研究, 24, 277-284.]

Chen F, Wang JM, Sun BG, Chen XM, Yang ZX, Duan ZY (2012) Relationships of plant species distribution in different strata of Pinus yunnanensis forest with landform and climatic factors. Chinese Journal of Ecology, 31, 1070-1076. (in Chinese with English abstract) [陈飞, 王健 敏, 孙宝刚, 陈晓鸣, 杨子祥, 段兆尧 (2012) 云南松林 不同层植物分布与地形、气候因子的关系. 生态学杂志, 31, 1070-1076.]

Chen XM, Lei YC, Zhang XQ, Jia HY (2012) Effects of sample sizes on accuracy and stability of maximum entropy model in predicting species distribution. Scientia Silvae Sinicae, 48(1), 53-59. (in Chinese with English abstract) [陈 新美, 雷渊才, 张雄清, 贾宏炎 (2012) 样本量对MaxEnt 模型预测物种分布精度和稳定性的影响. 林业科学, 48(1), 53-59.]

Dong XF (2017) Effect of topographic factors on the distribution of Manglietia insignis. Journal of Anhui Agricultural Sciences, 45(10), 162-163. (in Chinese with English abstract) [董学芬 (2017) 地形因素对红花木莲分布的影响. 安徽农业科学, 45(10), 162-163.]

Elith J, Phillips SJ, Hastie T, Dudík M, Chee YE, Yates CJ (2011) A statistical explanation of MaxEnt for ecologists. Diversity and Distributions, 17, 43-57.

Escalante T, Rodríguez-Tapia G, Linaje M, Illoldi-Rangel P, González-López R (2013) Identification of areas of endemism from species distribution models: Threshold selection and Nearctic mammals. Transaction Image Processing, 16, 5-17.

Getis A, Ord JK (1992) The analysis of spatial association by use of distance statistics. Geographical Analysis, 24, 189-206.
Hu X, Wu FC, Guo W, Liu N (2014) Identification of potential cultivation region for Santalum album in China by the MaxEnt ecologic niche model. Scientia Silvae Sinicae, 50(5), 27-33. (in Chinese with English abstract) [胡秀, 吴 福川, 郭微, 刘念 (2014) 基于MaxEnt生态学模型的檀香 在中国的潜在种植区预测. 林业科学, 50(5), 27-33.]

Jiang ZG (1996) Hoarding behavior of animal and its ecological functions. Chinese Journal of Zoology, (3), 47-49. (in Chinese) [蒋志刚 (1996) 动物拒食行为及其生态意义. 动物学杂志, (3), 47-49.]

Kunming Institute of Botany, Chinese Academy of Sciences (2006) Flora of Yunnan. Science Press, Beijing. (in Chinese) [中国科学院昆明植物研究所 (2006) 云南植物志. 科学 出版社, 北京.]

Levin SA (1992) The problem of pattern and scale in ecology: The Robert H. MacArthur Award lecture. Ecology, 73, 1943-1967.

Li HJ, Zhang ZB (2001) Relationship between animals and plant regeneration by seed. II. Seed predation, dispersal and burial by animals and relationship between animals and seedling establishment. Biodiversity Science, 9, 25-37. (in Chinese with English abstract) [李宏俊, 张知涁 (2001) 动 物与植物种子更新的关系. II. 动物对种子的捕食、扩散、 贮藏及与幼苗建成的关系. 生物多样性, 9, 25-37.]

Lin YP, Deng D, Lin WC, Lemmens R, Crossman ND, Henle K, Schmeller DS (2015) Uncertainty analysis of crowdsourced and professionally collected field data used in species distribution models of Taiwanese moths. Biological Conservation, 181, 102-110.

Manhães AP, Loyola R, Mazzochini GG, Ganade G, Oliveira-Filho AT, Carvalho AR (2018) Low-cost strategies for protecting ecosystem services and biodiversity. Biological Conservation, 217, 187-194.

Morales NS, Fernández IC, Bacagonzález V (2017) MaxEnt’s parameter configuration and small samples: Are we paying attention to recommendations? A systematic review. PeerJ, 5, e3093.

Myers N (1990) The biodiversity challenge: Expanded hot-spots analysis. Environmentalist, 10, 243 -256.

Nüchel J, Bøcher PK, Xiao W, Zhu AX, Svenning JC (2018) Snub-nosed monkeys (Rhinopithecus): Potential distribution and its implication for conservation. Biodiversity and Conservation, 27, 1517-1538.

Ord JK, Getis A (1995) Local spatial autocorrelation statistics: Distributional issues and an application. Geographical Analysis, 27, 286-306.

Phillips SJ, Anderson RP, Schapire RE (2006) Maximum entropy modeling of species geographic distributions. Ecological Modelling, 190, 231-259.

Phillips SJ, Dudík M, Schapire RE (2004) A Maximum Entropy Approach to Species Distribution Modeling. p. 83. Association for Computing Machinery, Banff.

Raes N, Roos MC, Slik JWF, van Loon EE, ter Steege H 
(2009) Botanical richness and endemicity patterns of Borneo derived from species distribution models. Ecography, 32, 180-192.

Raes N, Steege HT (2007) A null-model for significance testing of presence-only species distribution models. Ecography, 30, 727-736.

Scott JM, Csuti B, Jacobi JD, Estes JE (1987) Species richness. BioScience, 37, 782-788.

Sierra R, Campos F, Chamberlin J (2002) Assessing biodiversity conservation priorities: Ecosystem risk and representativeness in continental Ecuador. Landscape and Urban Planning, 59, 95-110.

Song W, Kim E, Lee D, Lee M, Jeon SW (2013) The sensitivity of species distribution modeling to scale differences. Ecological Modelling, 248, 113-118.

Tang JH, Cheng YX, Luo LZ, Zhang L, Jiang XF (2017) MaxEnt-based prediction of overwintering areas of Loxostege sticticalis in China under different climate change scenarios. Acta Ecologica Sinica, 37, 4852-4863. (in Chinese with English abstract) [唐继洪, 程云霞, 罗礼智, 张 蕾, 江幸福 (2017) 基于MaxEnt模型的不同气候变化情 景下我国草地螟越冬区预测. 生态学报, 37, 4852-4863.]

Tang ZY, Fang JY (2004) A review on the elevational patterns of plant species diversity. Biodiversity Science, 12, 20-28. (in Chinese with English abstract) [唐志尧, 方精云 (2004) 植物物种多样性的垂直分布格局. 生物多样性, 12, 20-28.]

Vaz ÚL, Cunha HF, Nabout JC (2015) Trends and biases in global scientific literature about ecological niche models. Brazilian Journal of Biology, 75(4), S17-S24.

Wang HL (2017) Protection and utilization of wild animals and plants in Lanping County. Journal of Green Science and Technology, (3), 90-91. (in Chinese) [汪海林 (2017) 兰坪 县野生动植物保护与利用对策. 绿色科技, (3), 90-91.]

Wiegand T, Gunatilleke S, Gunatilleke N (2007) Species associations in a heterogeneous Sri Lankan dipterocarp forest.
The American Naturalist, 170, E77.

Yang GB (2011) Ecological quality assessment of Yunling Nature Reserve in Lanping County of Yunnan Province. Journal of West China Forestry Science, 40(4), 48-53. (in Chinese with English abstract) [杨国斌 (2011) 兰坪云岭 省级自然保护区生态质量评价研究. 西部林业科学, 40(4), 48-53.]

Yang GB (2013) Lanping Yunling Nature Reserve. Yunnan Science and Technology Press, Kunming. (in Chinese) [杨 国斌 (2013) 兰坪云岭自然保护区. 云南科技出版社, 昆 明.]

Zhang L (2015a) Application of MaxEnt model in predicting the potential distribution of species. Bulletin of Biology, 50(11), 9-12. (in Chinese) [张路 (2015a) MaxEnt最大熵模 型在预测物种潜在分布范围方面的应用. 生物学通报, 50(11), 9-12.]

Zhang L (2015b) Prediction of potential distribution area of Euphorbia dentata in China based on MaxEnt model. Journal of Biosafety, 24, 194-200. (in Chinese with English abstract) [张路 (2015b) 基于MaxEnt模型预测齿裂大戟在 中国的潜在分布区. 生物安全学报, 24, 194-200.]

Zhao SQ, Fang JY, Lei GC (2000) Global 200: An approach to setting large-scale biodiversity conservation priorities. Chinese Biodiversity, 8, 435-440. (in Chinese with English abstract) [赵淑清, 方精云, 雷光春 (2000) 全球200: 确定 大尺度生物多样性优先保护的一种方法. 生物多样性, 8, 435-440.]

Zhu GP, Qiao HJ (2016) Effect of the MaxEnt model's complexity on the prediction of species potential distributions. Biodiversity Science, 24, 1189-1196. (in Chinese with English abstract) [朱耿平, 乔慧捷 (2016) MaxEnt模型复杂度 对物种潜在分布区预测的影响. 生物多样性, 24, 1189-1196.]

(责任编委：张大勇 责任编辑：黄祥忠、周玉荣)

\section{附录 Supplementary Material}

附录1 19个生物气候因子与 3 个地形因子的数据概况

Appendix 1 Data profiles of 19 bioclimatic variables and 3 terrain variables http://www.biodiversity-science.net/fileup/PDF/2018059-1.pdf 
庄鸿飞等. 基于最大熵模型的不同尺度物种分布概率优化热点分析：以红色木莲为例. 生物多样性, 2018, 26 (9): 931-940. http://www.biodiversity-science.net/CN/10.17520/biods.2018059

\section{附录1 19个生物气候因子与 3 个地形因子的数据概况}

Appendix 1 Data profiles of 19 bioclimatic variables and 3 terrain variables

\begin{tabular}{|c|c|c|}
\hline 变量 Variable & 描述 Description & 类别 Category \\
\hline b1 & 年均温 Annual mean temperature & 生物气候因子 Bioclimatic \\
\hline b2 & 昼夜温差月均值 Mean diurnal range (Mean of monthly (max. temp-min. temp)) & 生物气候因子 Bioclimatic \\
\hline b3 & 等温性 Isothermality $(\mathrm{b} 2 / \mathrm{b} 7)(\times 100)$ & 生物气候因子 Bioclimatic \\
\hline b4 & 温度季节性变化 Temperature seasonality (standard deviation × 100) & 生物气候因子 Bioclimatic \\
\hline b5 & 最暖月最高温度 Max temperature of warmest month & 生物气候因子 Bioclimatic \\
\hline b6 & 最冷月最低温度 Min temperature of coldest month & 生物气候因子 Bioclimatic \\
\hline b7 & 温度全年波动范围 Temperature annual range (b5-b6) & 生物气候因子 Bioclimatic \\
\hline b8 & 最湿季度平均温度 Mean temperature of wettest quarter & 生物气候因子 Bioclimatic \\
\hline b9 & 最干季度平均温度 Mean temperature of driest quarter & 生物气候因子 Bioclimatic \\
\hline b10 & 最暖季度平均温度 Mean temperature of warmest quarter & 生物气候因子 Bioclimatic \\
\hline b11 & 最冷季度平均温度 Mean temperature of coldest quarter & 生物气候因子 Bioclimatic \\
\hline b12 & 年降水量 Annual precipitation & 生物气候因子 Bioclimatic \\
\hline b13 & 最湿月降水量 Precipitation of wettest month & 生物气候因子 Bioclimatic \\
\hline b14 & 最干月降水量 Precipitation of driest month & 生物气候因子 Bioclimatic \\
\hline b15 & 降水量季节性变化 Precipitation seasonality (coefficient of variation) & 生物气候因子 Bioclimatic \\
\hline b16 & 最湿季度降水量 Precipitation of wettest quarter & 生物气候因子 Bioclimatic \\
\hline b17 & 最干季度降水量 Precipitation of driest quarter & 生物气候因子 Bioclimatic \\
\hline b18 & 最暖季度降水量 Precipitation of warmest quarter & 生物气候因子 Bioclimatic \\
\hline b19 & 最冷季度降水量 Precipitation of coldest quarter & 生物气候因子 Bioclimatic \\
\hline alt & 海拔 Altitude & 地形 Terrain \\
\hline slo & 坡度 Slope & 地形 Terrain \\
\hline asp & 坡向 Aspect & 地形 Terrain \\
\hline
\end{tabular}

生物气候因子数据来源于Worldclim, 分辨率为 $30 "$ 。地形数据来源于地理空间数据云, 分辨率为 $90 \mathrm{~m}$ 。

Data of Bioclimatic (resolution is 30") is from Worldclim. Data of terrain (resolution is $90 \mathrm{~m}$ ) is from GSCloud. 